\title{
Recent Results from T2K and Future Prospects
}

\author{
Konosuke Iwamoto, on behalf of the T2K Collaboration* \\ University of Rochester \\ E-mail: kiwamoto@pas.rochester.edu
}

\begin{abstract}
The T2K long-baseline neutrino oscillation experiment has been running in anti-neutrino mode since 2014 and recently released anti-neutrino oscillation results. These results have been updated with further data and now include the full three-flavor anti-neutrino oscillation analysis. We will present these new results and compare them with the neutrino oscillation results, giving the most sensitive comparison to date. The three-flavor neutrino and anti-neutrino results are used to obtain world-leading measurements of $\delta_{C P}, \theta_{23}$ and $\Delta m_{32}^{2}$.

The T2K long-baseline neutrino experiment is in the process of proposing a follow-up experiment, T2K-II, with higher beam intensity, upgraded detectors and improved sensitivity to neutrino properties. The anticipated sensitivity and reach of T2K-II will be discussed in this talk, as well as possible detector upgrades. In particular, the sensitivity should allow for a $3 \sigma$ discovery of CP violation in the case of maximum $\mathrm{CP}$ violation, after 10 years of data-taking.
\end{abstract}

38th International Conference on High Energy Physics

3-10 August 2016

Chicago, USA

${ }^{*}$ Speaker. 
Neutrino oscillation is a quantum mechanical phenomenon that causes neutrino flavor to change periodically as it travels through space. It is caused by the mixing between the flavor states and mass eigenstates which is expressed in a form of Pontecorvo-Maki-Nakagawa-Sakata (PMNS) matrix [1]. Neutrino oscillation probability is governed by the oscillation parameters, and the accelerator neutrino experiments utilize muon neutrino disappearance and electron neutrino appearance samples to measure the parameters. The disappearance probability can be expressed as functions of the mixing angle $\theta_{23}$ and mass difference term $\Delta m_{32}^{2}$, and as expressed in [2], the appearance probability depends on $\theta_{13}$ and $\mathrm{CP}$ violation phase $\delta_{C P}$ in addition to the previous parameters. While the oscillation probability of the muon neutrino disappearance is identical between the neutrino and anti-neutrino sectors in vacuum, they are different for the electron neutrino appearance due to the dependence on the $\mathrm{CP}$ odd phase $\sin \delta_{C P}$. This fundamental property of the neutrino oscillation makes the comparison between the neutrino and anti-neutrino results important to measure the $\delta_{C P}$ with high sensitivity.

In addition, the oscillation probability has the dependence on the ratio between the distance and energy of the traveling neutrino. This dependence allows one to design the experiment with careful considerations of the neutrino energy, E, spectrum and the baseline distance, L, to maximize the occurrence of the oscillation. The configurations of the neutrino kinematics allow the experiments whether the matter effect [3] on the neutrino oscillations to be negligible or not.

The T2K experiment has collected data to perform the three-flavor oscillation analyses considering both neutrino and anti-neutrino oscillation measurements with $\mathrm{L}=295 \mathrm{~km}$ and average $\mathrm{E}$ $=0.6 \mathrm{GeV}$ configurations. The recent $\mathrm{T} 2 \mathrm{~K}$ oscillation analysis result rejects the $\mathrm{CP}$ conservation by $90 \%$ confidence level. Furthermore, the T2K collaboration has a future prospect to perform upgrades in beam intensity, detector, and the analyses to collect sufficient data on the time scale of 2026 to "discover" the $\mathrm{CP}$ violation with $3 \sigma$ sensitivity.

\section{Detector Design and Analysis Method}

T2K (Tokai to Kamioka) [4] is a long baseline neutrino oscillation experiment conducted in Japan that utilizes the concept of near and far detectors to measure the neutrino interactions before and after the oscillations, respectively. The muon neutrino beam is prepared at the J-PARC accelerator and beam line in Tokai by $30 \mathrm{GeV}$ proton beam generated by the primary beam line main ring (MR) directed to the graphite target. The secondary pions generated by this collision are collected and focused by the set of magnetic horns located in the secondary beam line. The alteration of the polarity of the magnetic horns allows to select positively or negatively charged pions which decay into neutrinos or anti-neutrinos, respectively, to run the beam in either neutrino or anti-neutrino modes. The beam is then profiled and studied prior to the oscillations by the near detector complex ND280, and the neutrino interactions after the oscillations are studied by monitoring the Cherenkov radiations originated from them at the Super-Kamiokande (SK) far detector [5] in Kamioka, located $295 \mathrm{~km}$ away from Tokai.

The collaboration was established to achieve two major physics goals by studying the neutrino beam with the near and the far detectors: 1) to measure the oscillation parameters $\theta_{13}$ and $\delta_{C P}$ by observing the neutrino oscillation from muon to electron neutrino $\left(v_{\mu} \rightarrow v_{e}\right)$ and 2$)$ to make the precision measurements of $\theta_{23}$ and $\Delta m_{32}^{2}$ from the $v_{\mu}$ disappearance from the beam. In order 
to maximize the probability of the muon neutrino oscillation at SK far detector, T2K uses the off-axis method to create a narrow-band neutrino beam which the energy peaks around $0.6 \mathrm{GeV}$. The ND280 measurements combined with the external experimental data provide the neutrino flux predictions and interaction cross section models to perform the sophisticated prediction of the event composition at the SK detector with constrained systematic uncertainties. The SK detector efficiency is evaluated using the control samples such as SK atmospheric neutrino data and $\pi^{0}$ control samples.

The T2K oscillation analyses use measurements in both neutrino and anti-neutrino modes simultaneously to perform the full joint data fit. The maximal likelihood method is used to calculate the sets of best fit oscillation parameters with consideration of normal or inverted hierarchy. The likelihood calculations consider number of observed neutrino or anti-neutrino candidate events, shape of the kinematic distributions, and the systematic uncertainties that correspond to the samples interested $\left(v_{\mu} / \bar{v}_{\mu}\right.$ disappearance or $v_{e} / \bar{v}_{e}$ appearance events) [6] .

\section{Statistical Update and Latest Analysis Results}

As of May 27, 2016, the T2K experiment has collected $7.482 \times 10^{20}$ and $7.471 \times 10^{20}$ protons on target (POT) in neutrino and anti-neutrino modes, respectively. Figure 1 shows the summary of the POT accumulated by $\mathrm{T} 2 \mathrm{~K}$ in neutrino and anti-neutrino modes and the corresponding beam power. Using the data set available, we performed three major studies. The full joint data fit is performed to calculate the best fit oscillation parameters $\delta_{C P}, \theta_{23}$ and $\Delta m_{32}^{2}$. The $v_{\mu}$ disappearance results in neutrino and anti-neutrino modes are compared to investigate the evidence of CPT violation or non-standard neutrino interactions; this study was performed with $7.002 \times 10^{20}$ and $7.471 \times 10^{20}$ POT in neutrino and anti-neutrino modes, respectively. In addition, we introduce the selection of additional $v_{e}$ charged current $(\mathrm{CC})$ resonance $1 \pi^{+}$appearance sample in $7.482 \times 10^{20}$ POT in neutrino mode by modifying the current $v_{e}$ appearance selection criteria.

\subsection{Full Joint Fit Oscillation Analysis}

The full data set with neutrino and anti-neutrino samples are used to perform the oscillation parameter fits to measure $\delta_{C P}, \theta_{23}$ and $\Delta m_{32}^{2}$. Among the accumulated data set, we have obtained $135 v_{\mu}$ disappearance and $32 v_{e}$ appearance candidate events in neutrino mode and $66 \bar{v}_{\mu}$ disappearance and $4 \bar{v}_{e}$ appearance candidate events in anti-neutrino mode samples. Figure 2 shows the spectra of the reconstructed kinematic variables for each sample category. The comparisons between the best fit and the unoscillated predictions show the expected decreases in the event rates at the reconstructed energy peak at $0.6 \mathrm{MeV}$ in both $v_{\mu}$ and $\bar{v}_{\mu}$ disappearance samples.

Data fit results of the disappearance parameters, $\theta_{23}$ and $\Delta m_{32}^{2}$, are shown on Figure 3. The T2K fit result obtain $\sin ^{2} \theta_{23}=0.532_{-0.068}^{+0.046}\left(0.534_{-0.066}^{+0.043}\right)$ and $\Delta m_{32}^{2}=2.545_{-0.084}^{+0.081}\left(2.510_{-0.083}^{+0.081}\right) \times$ $10^{-3} \mathrm{eV}^{2}$ with normal (inverted) mass hierarchy hypothesis. The T2K best fit point and the confidence level contours for the disappearance parameters are compared with the external experimental results: IceCube, NOva, MINOS+, and Super-K. The T2K fit result prefers maximal $\theta_{23}$ mixing; however, despite the fact that they are in agreement within $68 \%$ and $90 \%$ confidence levels, it is worthwhile to emphasize that the shapes of the confidence level contours are significantly different between the $\mathrm{T} 2 \mathrm{~K}$ and NOvA results. 


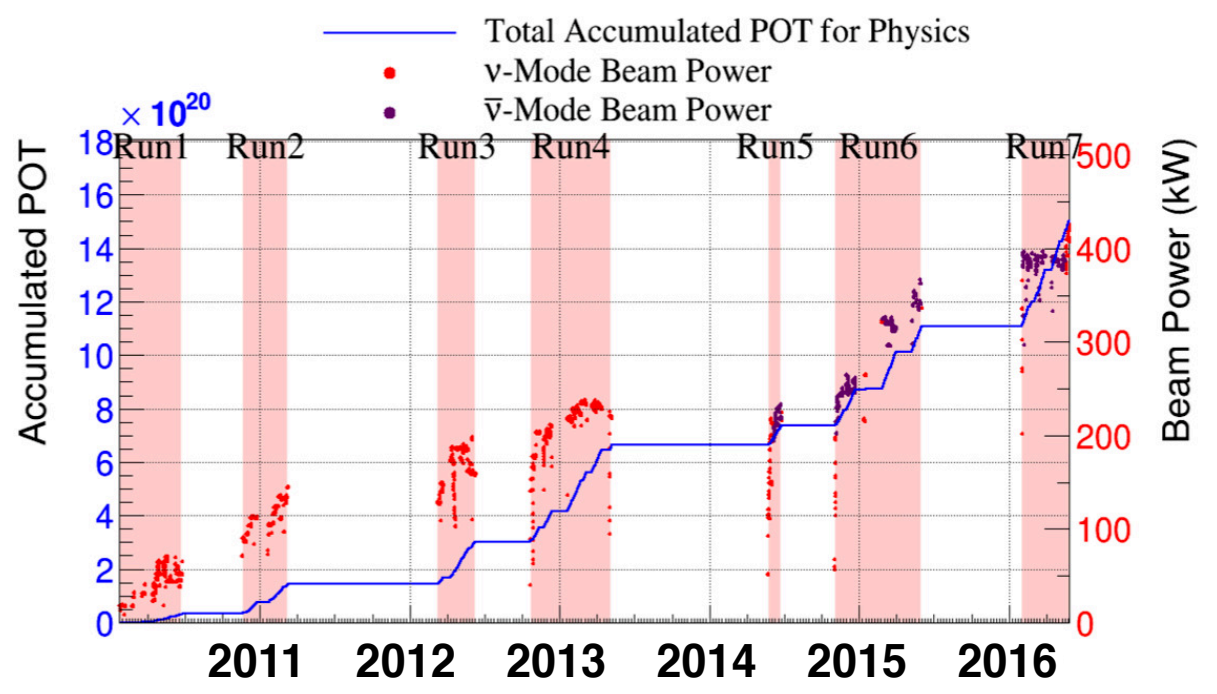

Figure 1: Summary of the POT accumulated by T2K in neutrino and anti-neutrino modes and the corresponding beam power. The blue line shows the total accumulated POT for physics. Red and purple dots show the beam power in neutrino and anti-neutrino modes, respectively.

$\mathrm{CP}$ violating phase $\delta_{C P}$ is studied with the full joint fit analysis. Table 1 shows the expected event rates of the $v_{e}$ and $\bar{v}_{e}$ appearance samples for different $\delta_{C P}$ values. Our simulations predict the enhancement and suppression of the appearance event rates in neutrino and anti-neutrino modes due to the $\mathrm{CP}$ odd phase $\sin \delta_{C P}$. Figure 4 shows the data fit results in $\delta_{C P}-\sin ^{2} \theta_{13}$ space. The confidence level contours and the best fit points are calculated with and without the constraint on the $\sin \theta_{13}$ region by the reactor experiment results $\left(\sin ^{2} 2 \theta_{13}=0.085 \pm 0.005\right)$. The T2K-only result shows the consistent result with the reactor experiment measurements, with the preference on the maximally $\mathrm{CP}$ violated region at $\delta_{C P} \sim-\frac{\pi}{2}$.

\begin{tabular}{|c|c|c|c|c|c|}
\hline Sample & $\begin{array}{c}\delta_{C P}=-\frac{\pi}{2} \\
\text { Normal Hierarchy }\end{array}$ & $\begin{array}{c}\delta_{C P}=0 \\
\text { Normal Hierarchy }\end{array}$ & $\begin{array}{c}\delta_{C P}=+\frac{\pi}{2} \\
\text { Normal Hierarchy }\end{array}$ & $\begin{array}{c}\delta_{C P}=\pi \\
\text { Normal Hierarchy }\end{array}$ & Observed \\
\hline$v_{e}$ & 28.7 & 24.2 & 19.6 & 24.1 & 32 \\
\hline $\bar{v}_{e}$ & 6.0 & 6.9 & 7.7 & 6.8 & 4 \\
\hline
\end{tabular}

Table 1: Comparison of the expected total number of events in each sample at different $\delta_{C P}$ values and the observed candidates.

The T2K measurement of the $\delta_{C P}$ confidence level is studied with the reactor constraint and compared with the simulations on Figure 5. The comparison of the expected sensitivities between the $7.002 \times 10^{20}$ (red) and $7.482 \times 10^{20}$ (black) POT in neutrino mode are shown to provide the effect of the improvement in the data fit with the extra statistics. The $\Delta \chi^{2}$ distributions have different shapes with higher values at the peak at $\delta_{C P} \frac{\pi}{2}$ between the expected sensitivity and the measurement which arise from the fact that more (less) events are observed in data for $v_{e}\left(\bar{v}_{e}\right)$ appearance sample.

Since there is a physical boundary at $\delta_{C P}= \pm \frac{\pi}{2}$, in order to properly evaluate the confidence interval of the $\delta_{C P}$, the critical $\Delta \chi^{2}$ values are calculated using Feldman-Cousins method [7]. The studies have shown that the $\delta_{C P}=[-3.13 ;-0.39]$ and $[-2.09 ;-0.74]$ with $90 \%$ confidence level 

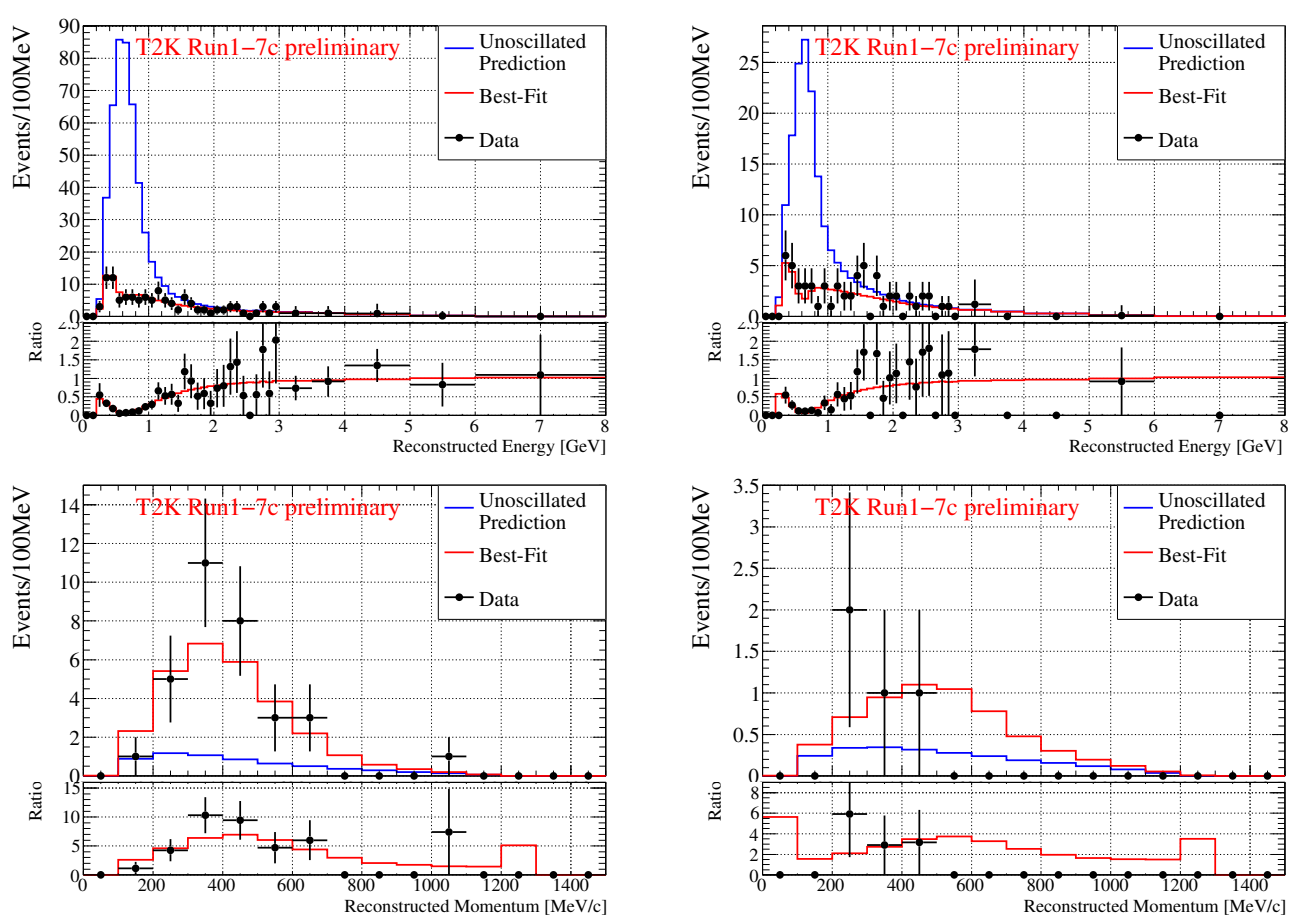

Figure 2: Spectra of the reconstructed kinematic variables predicted with the unoscillated prediction (blue) and the best-fit oscillation parameters (red) and the event candidates from the data (black). Reconstructed neutrino energy spectrum is used for $v_{\mu}$ (top left) and $\bar{v}_{\mu}$ (top right) disappearance samples and reconstructed lepton momentum spectrum is used for $v_{e}$ (bottom left) and $\bar{v}_{e}$ (bottom right) appearance samples. The distributions under the spectra are the ratio between the best fit and unoscillated spectra.

in normal and inverted hiearchy hypotheses, respectively. The T2K measurement rejects the CP conservation in neutrino oscillation for both mass hierarchy hypotheses with $90 \%$ confidence level.

\section{$2.2 v_{\mu}$ and $\bar{v}_{\mu}$ Disappearance Comparison}

Since the $v_{\mu}$ and $\bar{v}_{\mu}$ disappearance probabilities are identical at leading order (neglecting matter effects small for $\mathrm{T} 2 \mathrm{~K}$ baseline) $\sin ^{2} 2 \theta_{23}$, inconsistent measurements in the oscillation parameters between the $v_{\mu}$ and $\bar{v}_{\mu}$ disappearance analyses could be an evidence of the CPT violation or non-standard neutrino interactions. The "CPT test" is performed and shown on Figure 6 by comparing the sensitivities in $\Delta m_{32}^{2}-\sin ^{2} \theta_{23}$ space. The POT accumulation of $7.002 \times 10^{20}$ and $7.471 \times 10^{20}$ in neutrino and anti-neutrino modes, respectively, is used to perform the studies. 125 and 66 candidates are observed in neutrino and anti-neutrino modes, respectively. With both hierarchy cases, the confidence level contours show good agreement between the $v_{\mu}$ and $\bar{v}_{\mu}$ measurements. Therefore, no hint of CPT violation or non-standard neutrino interactions is observed.

\subsection{Selection of Additional $v_{e} \mathrm{CC} 1 \pi^{+}$Appearance Sample}

The study is performed to investigate non-charge-current-quasielastic scattering (CCQE) signal events to increase the statistics available for the $v_{e}$ appearance analysis. In particular, we introduce the selection of additional $v_{e} \mathrm{CC} 1 \pi^{+}$appearance sample by modifying the current $v_{e}$ appearance selection criteria. As explained in [6], the current T2K $v_{e}$ appearance selection criteria 


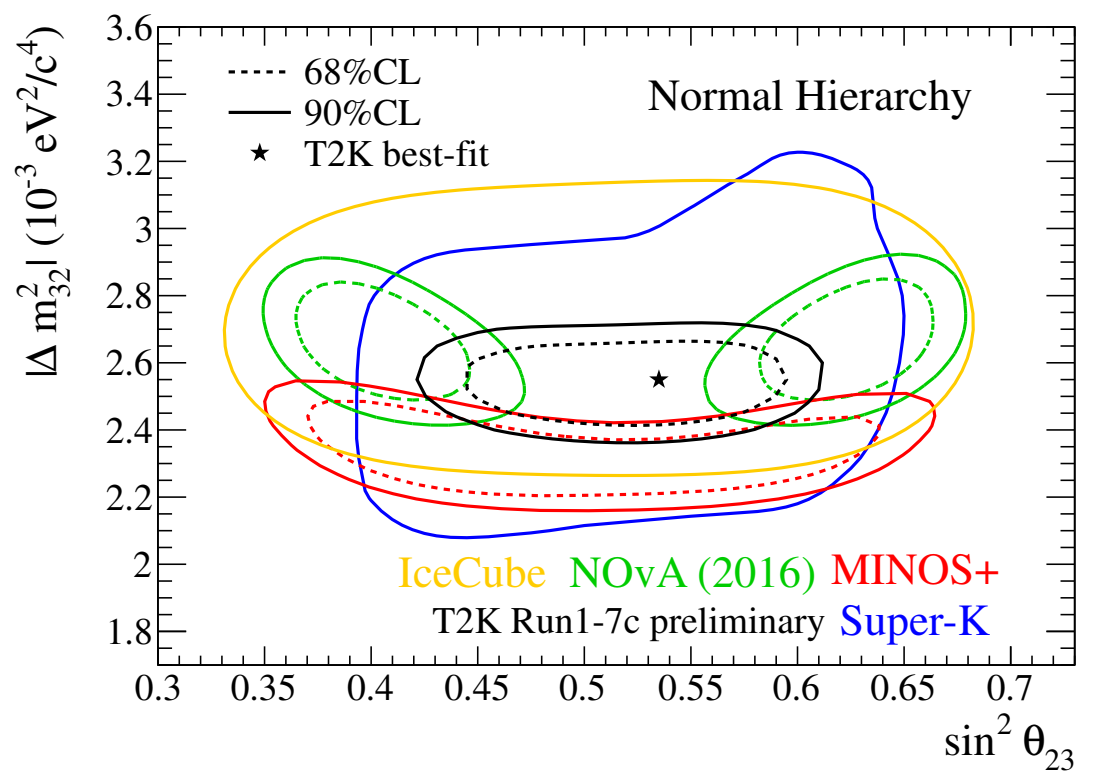

Figure 3: Best fit point (black dot) and the confidence level contours (black lines) for $\Delta m_{32}^{2}-\sin ^{2} \theta_{23}$ space. External results from IceCube (yellow), NOvA (green), MINOS+ (red), and Super-K (blue) are shown for the comparison.
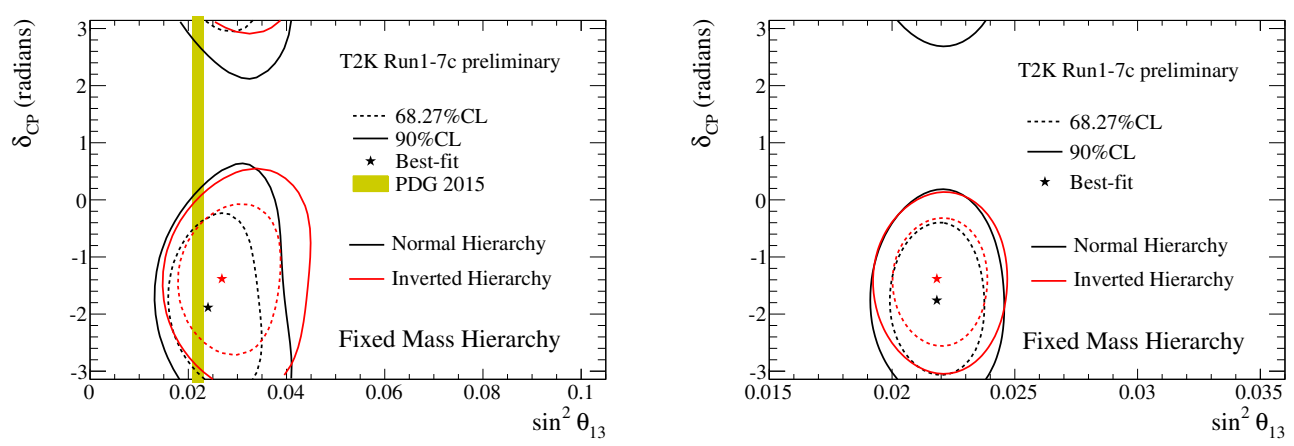

Figure 4: Best fit point (dots) and the confidence level contours (lines) for $\delta_{C P}-\sin ^{2} \theta_{13}$ space. The best fit with the normal (black) and inverted (red) hierarchy hypotheses are shown separately. Left contours are calculated using only the T2K data; the yellow shaded $\sin ^{2} \theta_{13}$ region shows the results obtained by the reactor experiment $\left(\sin ^{2} 2 \theta_{13}=0.085 \pm 0.005\right)$. Right contours are calculated with the $\mathrm{T} 2 \mathrm{~K}$ data constrained by the reactor experiment results.

extract the event candidates with single-ring, electron-like Cherenkov pattern without the delayed detection of the decay electron according to the SK event reconstruction algorithm. They are designed to select $v_{e}$ CCQE events as the main signal interaction mode. In order to investigate for the additional statistics, single-ring, electron-like events with one decay electron are selected and studied as the $v_{e}$ CC resonance $1 \pi^{+}$signal candidates. This way, we select $\pi^{+}$interactions below the Cherenkov threshold with the pion that decays into muons which decays into the electrons.

In the current $v_{e}$ appearance analysis, the reconstructed neutrino energy is calculated consid- 

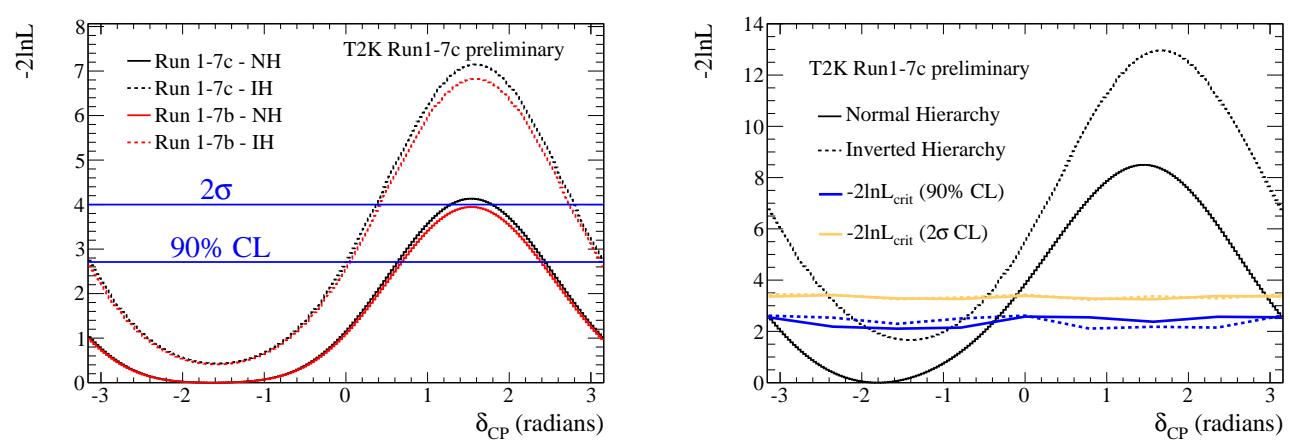

Figure 5: $1 \mathrm{D} \Delta \chi^{2}$ distributions as a function of $\delta_{c p}$ obtained with the constraint on the $\sin ^{2} \theta_{13}$ with the reactor experiment results. The left distributions show the expected sensitivity improvement from the $7.002 \times 10^{20}$ (red) to $7.482 \times 10^{20}$ (black) POT in neutrino mode. The right distributions show the data fit result with critical $\Delta \chi^{2}$ values for $90 \%$ (blue) and $2 \sigma$ (yellow) confidence levels obtained by the FeldmanCousins method.
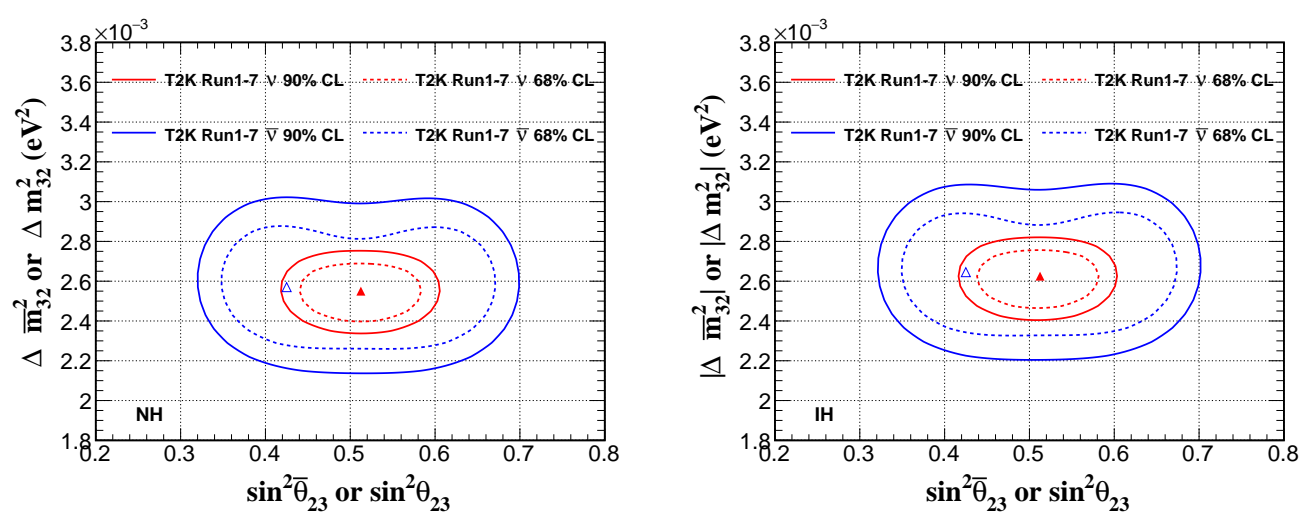

Figure 6: Best fit points (dots) and the confidence level contours (lines) for $\Delta m_{32}^{2}-\sin ^{2} \theta_{23}$ space calculated with the $v_{\mu}$ (red) and $\bar{v}_{\mu}$ (blue) disappearance fits performed separately. The left and right contours are obtained with the normal and inverted hierarchy hypotheses, respectively. This study was performed with $7.002 \times 10^{20}$ and $7.471 \times 10^{20}$ POT data in neutrino and anti-neutrino modes, respectively.

ering the elastic scattering from a nucleon in a potential well:

$$
E_{v_{e}}^{\mathrm{Rec}} \mathrm{CCQE}=\frac{2\left(M_{n}-V_{\mathrm{nuc}}\right) * E_{e}+M_{p}^{2}-\left(M_{n}-V_{\mathrm{nuc}}\right)^{2}-M_{e}^{2}}{2\left(\left(M_{n}-V_{\mathrm{nuc}}\right)-E_{e}+p_{e} \cos \theta_{e}\right)}
$$

where $M_{p}=$ proton mass, $M_{n}=$ neutron mass, $M_{e}=$ electron mass, $V_{n u c}=$ nuclear binding potential $(27.0 \mathrm{MeV}), E_{e}=$ reconstructed electron energy, $p_{e}=$ reconstructed electron momentum, and $\theta_{e}=$ angle between the beam and the electron.

Since we consider the delta resonance decaying into pion, it is inaccurate to use the energy reconstruction stated above. While there are several methods to reconstruct the neutrino energy for $v_{e} \mathrm{CC} 1 \pi^{+}$interaction, we consider the energy reconstruction formula by identifying the outgoing baryon as a $\Delta^{++}$resonance, instead of a proton, which is produced by the neutrino reaction on the 
free protons in the SK water:

$$
E_{v_{e} \mathrm{CC} \Delta}^{\mathrm{Rec}}=\frac{2 M_{p} E_{e}+M_{\Delta^{++}}^{2}-M_{p}^{2}-M_{e}^{2}}{2\left(M_{p}-E_{e}+p_{e} \cos \theta_{e}\right)}
$$

where $M_{\Delta^{++}}=\Delta^{++}$resonance with fixed mass of $1232.0 \mathrm{MeV} / \mathrm{c}^{2}$.

Table 2 shows the expected number of events in the $v_{e} \mathrm{CC} 1 \pi^{+}$appearance sample with different $\delta_{C P}$ values. Similarly with the $v_{e} \mathrm{CCQE}$ appearance sample, the enhancement and suppression of the event rates can be observed for different $\delta_{C P}$ values. The prediction shows that the inclusion of $v_{e} \mathrm{CC} 1 \pi^{+}$sample introduces approximately $11 \%$ more events with an assumption of the maximal CP violation at $\delta_{c p}=-\frac{\pi}{2}$.

Figure 7 shows the expected and measured event candidates of the $v_{e} \mathrm{CC} 1 \pi^{+}$appearance selection. Number of $v_{e}$ candidates observed in $7.482 \times 10^{20}$ POT in neutrino mode are binned as a function of number of decay electron to provide the comparison between the $v_{e}$ CCQE and $v_{e} \mathrm{CC} 1 \pi^{+}$selections; $5 v_{e} \mathrm{CC} 1 \pi^{+}$candidates are observed. The reconstructed neutrino energy spectrum of the $v_{e} \mathrm{CC} 1 \pi^{+}$sample is studied as well; despite the fact that the statistics is low in this sample category, the distributions of the $v_{e} \mathrm{CC} 1 \pi^{+}$candidates agree with the predicted number of event candidates and the spectrum. The oscillation analysis with inclusion of the $v_{e} \mathrm{CC} 1 \pi^{+}$sample is in progress.

\begin{tabular}{|c|c|c|c|c|c|}
\hline Sample & $\begin{array}{c}\delta_{C P}=-\frac{\pi}{2} \\
\text { Normal Hierarchy }\end{array}$ & $\begin{array}{c}\delta_{C P}=0 \\
\text { Normal Hierarchy }\end{array}$ & $\begin{array}{c}\delta_{C P}=+\frac{\pi}{2} \\
\text { Normal Hierarchy }\end{array}$ & $\begin{array}{c}\delta_{C P}=\pi \\
\text { Normal Hierarchy }\end{array}$ & Observed \\
\hline$v_{e} \mathrm{CC} 1 \pi^{+}$ & 3.1 & 2.8 & 2.3 & 2.7 & 5 \\
\hline
\end{tabular}

Table 2: Comparison of the expected total number of events in $v_{e} \mathrm{CC} 1 \pi^{+}$sample at different $\delta_{C P}$ values and the observed candidates.
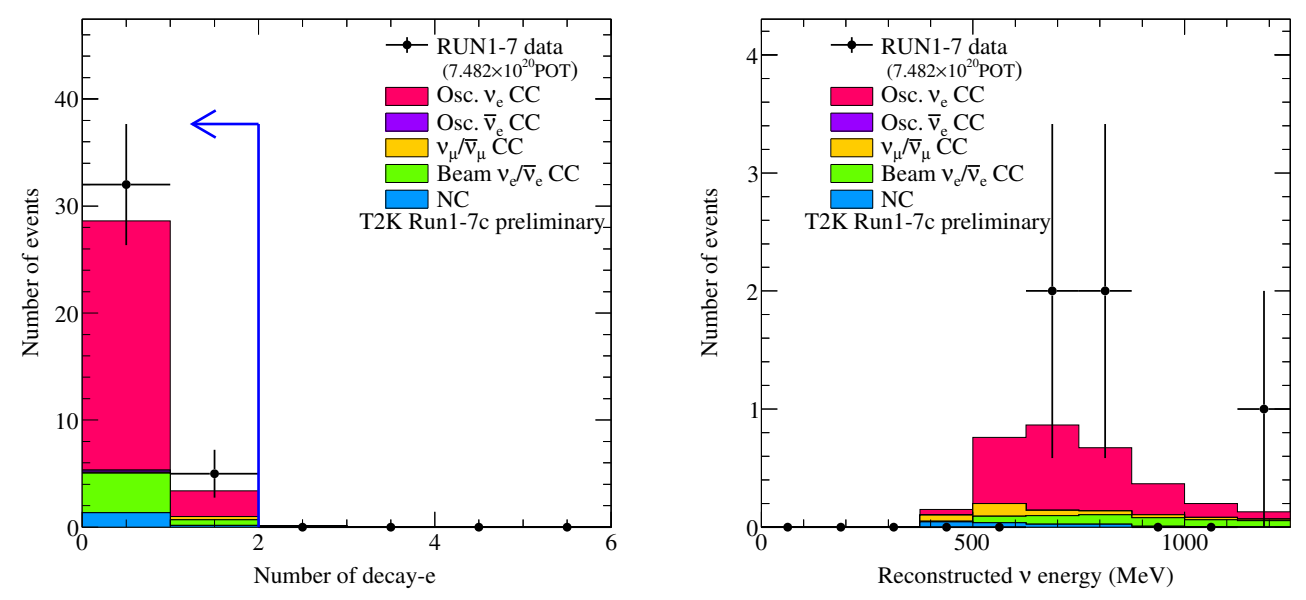

Figure 7: Expected (histograms) and measured (black dots) event candidates of the $v_{e}$ appearance selections. The left distribution shows the number of $v_{e} \mathrm{CC} 0 \pi$ and $v_{e} \mathrm{CC} 1 \pi^{+}$candidates as a function of number of decay electrons. The right distribution shows the reconstructed neutrino energy spectrum of the $v_{e} \mathrm{CC} 1 \pi^{+}$ sample. 


\section{A Proposed Extension of the T2K Experiment}

As of August 2016, T2K is approved to continue the run to accumulate $7.8 \times 10^{21} \mathrm{POT}$, which is expected around 2020. The $\mathrm{T} 2 \mathrm{~K}$ collaboration proposes further extension of the $\mathrm{T} 2 \mathrm{~K}$ run to 2026 called T2K-II, which anticipates collect sufficient statistics to "discover" CP violation with sensitivity greater than $3 \sigma$ if the $\delta_{C P}$ is maximally violated. Figure 8 shows the expected J-PARC MR beam power and the POT accumulation up to 2026 Japanese Fiscal Year (JFY). In order to accumulate the expected POT of $20 \times 10^{21}$ by $2026 \mathrm{JFY}$, the accelerator and MR beam power need to be upgraded to reach $1.3 \mathrm{MW}$ from the current beam power of $420 \mathrm{~kW}$. Various aspects of the T2K experiment need to be upgraded accordingly as well. Increase in an effective POT by $\sim 50 \%$ due to the upgrades such as the electromagnetic horn current upgrade from present 250kA to $320 \mathrm{kA}$, the near detector upgrade, and the analysis improvement are stated [8].

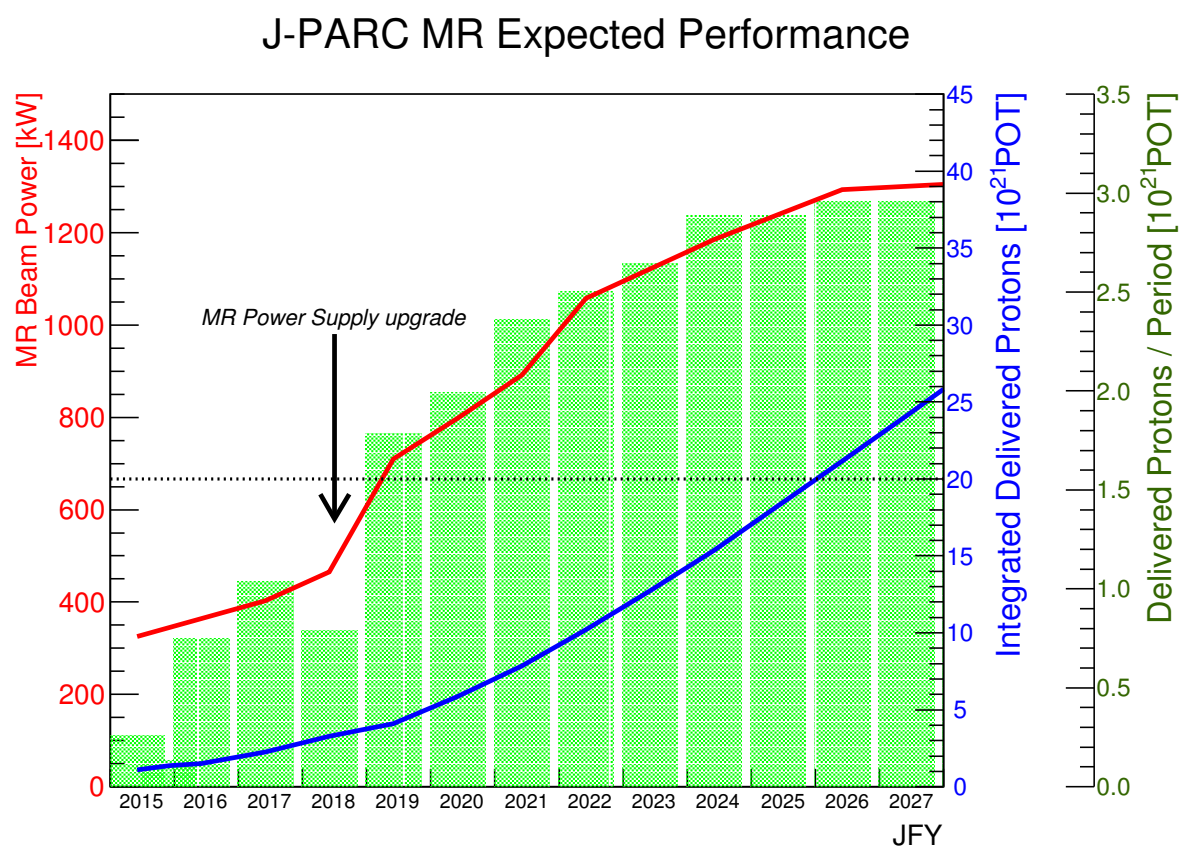

Figure 8: Expected J-PARC MR beam power and the POT accumulation as a function of calendar year.

Figure 9 shows examples of the sensitivity improvement anticipated by the full T2K-II exposure of $20 \times 10^{21}$ POT. Expected sensitivities to $\delta_{C P}$ with known and unknown mass hierarchy and $\Delta m_{32}^{2}-\sin ^{2} \theta_{23}$ space assuming $\theta_{23}$ at lower octant $\left(\sin ^{2} \theta_{23}=0.43\right)$ are studied with the T2K systematic errors from 2016. The sensitivities with the currently approved $7.8 \times 10^{21}$ POT exposure are prepared as the comparisons. The full T2K-II exposure achieve greater than $3 \sigma$ sensitivity to $\delta_{C P}$ and precise measurement of $\theta_{23}$ with resolution of $1.7^{\circ}$.

\section{Conclusion}

The T2K collaboration has obtained the measurements of $\delta_{C P}, \theta_{23}$ and $\Delta m_{32}^{2}$ by based on the three-flavor neutrino and anti-neutrino oscillation results. The full data set of $7.482 \times 10^{20}$ 

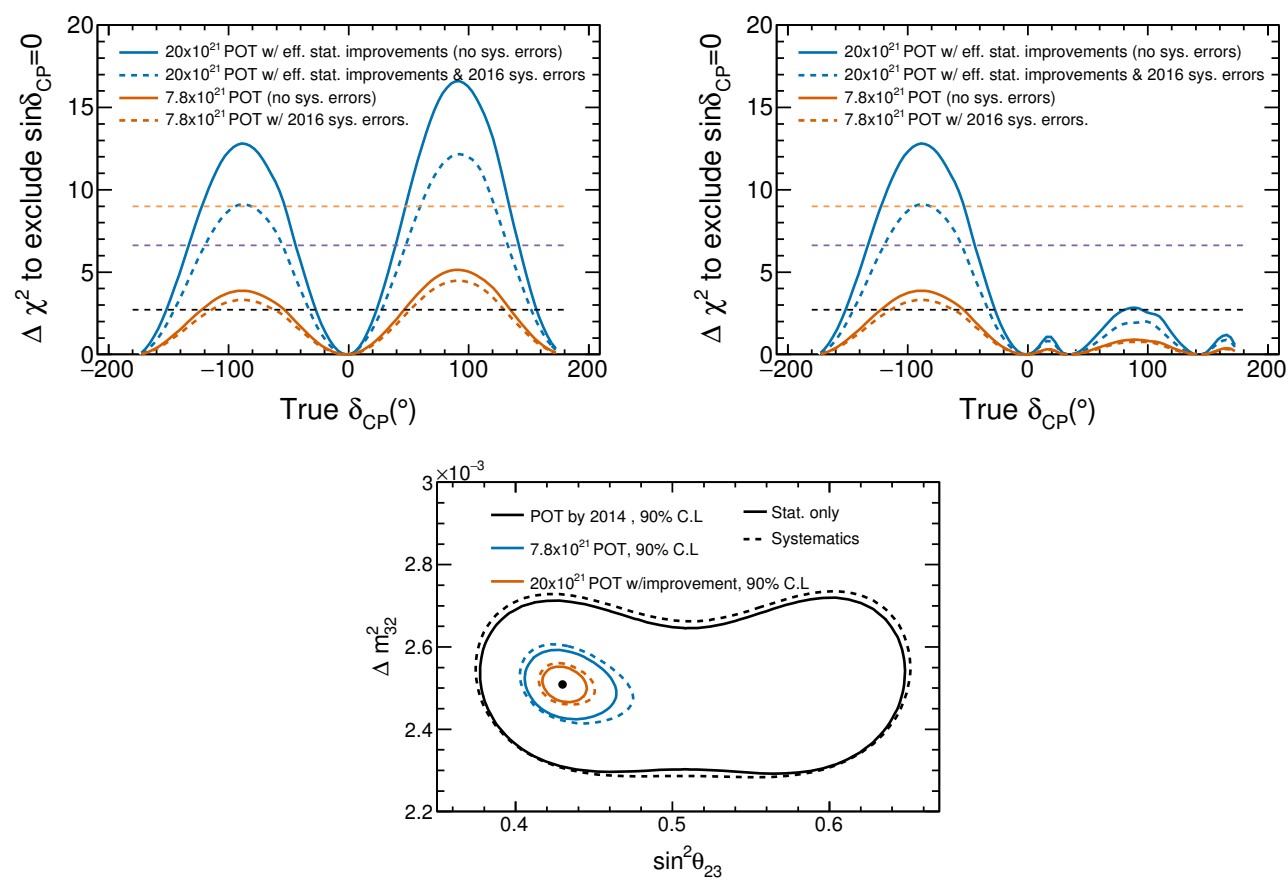

Figure 9: Sensitivity studies performed with the expected POT exposure of T2K-II with 2016 T2K systematic errors. Top distributions show the sensitivity to $\delta_{C P}$ assuming the known (left) and unknown (right) mass hierarchies. The bottom contour shows the sensitivity to $\Delta m_{32}^{2}-\sin ^{2} \theta_{23}$ space assuming true $\sin ^{2} \theta_{23}=0.43$.

and $7.471 \times 10^{20}$ POT in neutrino and anti-neutrino modes, respectively, is collected by May 27, 2016. The T2K measurements are consistent with maximal $\theta_{23}$ mixing and maximal $\mathrm{CP}$ violating phase at $\delta_{C P} \sim-\frac{\pi}{2}$ in normal hierarchy. The $\delta_{C P}$ result rejects the CP conservation at $90 \%$ confidence level in both normal and inverted hierarchy hypotheses: $\delta_{C P}=[-3.13 ;-0.39]$ (normal) and $[-2.09 ;-0.74]$ (inverted). The comparison between the neutrino and anti-neutrino results shows no hint of CPT violation or non-standard neutrino interactions. The non-CCQE signal events with a decay electron are investigated as a new sample set and studied to increase the statistics available for the $v_{e}$ appearance analysis; the oscillation analysis with inclusion of such sample is in progress.

The proposal of T2K-II to extend the T2K run to 2026 is expected to achieve the full exposure of $20 \times 10^{20}$ POT. Upgrades of the beam line, detectors, and analyses are required accomplish to reach this expectation with effective statistical increase by $\sim 50 \%$. The T2K-II sensitivity allows greater than $3 \sigma$ sensitivity to "discover" $\mathrm{CP}$ violation in the case of maximum $\mathrm{CP}$ violation.

\section{References}

[1] Z. Maki., M. Nakagawa, S. Sakata. Remarks on the Unified Model of Elementary Particles. Progress of Theoretical Physics 28 (1962) 870.

[2] M. Freund. Analytic Approximations for Three Neutrino Oscillation Parameters and Probabilities in Matter. Phys. Rev. D 64 (2001) 053003.

[3] L. Wolfenstein. Neutrino Oscillations in Matter. Phys. Rev. D 17 (1978) 2369.

[4] T2K Collaboration. The T2K Experiment. 8 Jun 2011. arXiv:1106.1238v2. 
[5] S. Fukuda, et. al. The Super-Kamiokande detector. Nuclear Instruments and Methods in Physics Research A501 (April 2003) 418-462. doi:10.1016/S0168-9002(03)00425-X.

[6] T2K Collaboration. Measurements of neutrino oscillation in appearance and disappearance channels by the T2K experiment with 6.6E20 protons on target. 5 Feb 2015. arXiv:1502.01550v1.

[7] G.J. Feldman and R.D. Cousins. Unified Approach to the Classical Statistical Analysis of Small Signals. Phys. Rev. D 57, 3873 (1998).

[8] K. Abe et al.Proposal for an Extended Run of T2K to $20 \times 10^{21}$ POT. arXiv:1609.04111 [hep-ex]. 